\title{
The tolerance for visual feedback distortions in a virtual environment
}

\author{
Yoky Matsuoka $^{\mathrm{a}, *}$, Sonya J. Allin ${ }^{\mathrm{b}}$, Roberta L. Klatzky ${ }^{\mathrm{c}}$ \\ ${ }^{a}$ Robotics Institute and Mechanical Engineering, Carnegie Mellon University, 5000 Forbes Avenue, NSH3207, Pittsburgh, PA 15213, USA \\ ${ }^{\mathrm{b}}$ Human Computer Interface Institute, Carnegie Mellon University, Pittsburgh, PA 15213, USA \\ ${ }^{\mathrm{c}}$ Department of Psychology, Carnegie Mellon University, Pittsburgh, PA 15213, USA
}

Received 9 July 2002; accepted 4 September 2002

\begin{abstract}
We are interested in using a virtual environment with a robotic device to extend the strength and mobility of people recovering from strokes by steering them beyond what they had thought they were capable of doing. Previously, we identified just noticeable differences (JND) of a finger's force production and position displacement in a virtual environment. In this paper, we extend this investigation by identifying peoples' tolerance for distortions of visual representations of force production and positional displacement in a virtual environment. We determined that subjects are not capable of reliably detecting inaccuracies in visual representation until there is $36 \%$ distortion. This discrepancy between actual and perceived movements is significantly larger than the JNDs reported in the past, indicating that a virtual robotic environment could be a valuable tool for steering actual movements further away from perceived movements. We believe this distorted condition may allow people recovering from strokes, even those who have perceptual or cognitive deficits, to rehabilitate with greater ease.
\end{abstract}

(C) 2002 Elsevier Science Inc. All rights reserved.

Keywords: Robotic rehabilitation; Virtual environment; Just noticeable difference; Feedback distortion

\section{Introduction}

The motor impairment of people recovering from strokes is often localized to one side of the body and to one area in particular, such as the arm or the hand. Motor recovery occurs as the nervous system rewires its neural circuits to represent lost functions at a new neural location. Recently, novel rehabilitation techniques such as constraint-induced therapy, biofeedback therapy, and robot-assisted therapy have been employed [1-3]. Specifically, robotic techniques enable the precise recording of movements and variable force applications to an affected limb, making it an effective strategy for motor rehabilitation. According to recent studies in robotics, robot-assisted stroke rehabilitation enhances arm movement recovery [2]. Moreover, robot-assisted rehabilitation improves patients' mobility and strength to the point where it is equal to or greater than that which is achieved by humanassisted therapy [3-5]. However, none of the currently

\footnotetext{
* Corresponding author. Tel.: +1-412-268-8127; fax: +1-412-2686436.

E-mail address: yoky@cs.cmu.edu (Y. Matsuoka).
}

available systems addresses patients' cognitive or perceptive deficits, which may provide patients with a false perception of their own ability. This false perception has been implicated as a potential factor in inhibiting motor recovery in rehabilitation technique that exists to date. To overcome this issue, we plan to create a perceptual motor rehabilitation technique using a virtual environment with a robotic device. This technique will make use of the perceptual gap that we will produce between the virtual and the real environments by distorting the virtual feedback by an imperceptible amount, and the lowest bound of this imperceptible distortion is determined by the just noticeable differences (JND) in force and position. This technique will extend current robotic rehabilitation techniques by creating an environment where patients can improve their mobility and strength without conscious effort, thereby addressing the needs of patients who may have false perceptions about their abilities.

A JND is defined as the percentage of increase in stimulus that is required to reliably distinguish two stimuli. Much prior research has focused on JNDs for force in human subjects, but none, to our knowledge, has tailored its findings to the rehabilitation domain. JNDs for lifting 2or 32-oz. weights by hand and arm were determined to be 
roughly $10 \%[6,7]$. JNDs for a force-matching task about the elbow were determined to be between $5 \%$ and $9 \%$ [8] and between $5 \%$ and $10 \%$ for pinching motions between the finger and the thumb with a constant resisting force [9]. The pinching JND was found to be relatively constant over base forces ranging between 2.5 and $10 \mathrm{~N}$.

In previous research, we conducted experiments with healthy subjects to derive JNDs for both force production and positional displacement using the same virtual environment with the same robotic device that we used in the experiment that is described in this paper (preliminary results in Ref. [10]). Using this environment, we derived JNDs while subjects moved their index fingers against resistive force produced by the robotic device. For a force JND, subjects were asked to sample pairs of forces by pressing their index fingers against the force produced by the robotic device while their fingers were occluded, and visual feedback of the force was provided. The robotic device produced force between 1.8 and $4.0 \mathrm{~N}$. Our results revealed an average JND of $14.4 \%$. We also conducted a similar experiment for a positional displacement JND, and it was calculated to be $18.0 \%$.

In this paper, we report the extent to which patients tolerate distortions in visual representations of force and position in a virtual environment. We hypothesized that force and position JNDs could be extended when subjects were given visual guidance that indicated that their force and position were different from their actual force and position. Because proprioceptive sensors are duller than visual sensors, small deviations between an actual position (assuming it is occluded) and the one displayed on a computer screen are not perceived. If visual feedback distortions extend the force and position of JNDs, then we may be able to extend the ability of stroke patients without their conscious effort.

\section{Methods}

\subsection{Apparatus}

We used a commercially available robotic device called PHANToM Premium 1.5 (Sensable Technologies, Cambridge, MA) to provide force feedback in virtual environments. This machine has three actuated and three passive degrees of freedom, creating a workspace of $19.5 \times$ $27.0 \times 37.5 \mathrm{~cm}$ and a maximum force that can be exerted of $8.5 \mathrm{~N}$. Although these workspace and force are small, they are large enough for hand/wrist level training while not having the potential to injure users. We decided to use this machine both because it works in a three-dimensional environment (as opposed to a two-dimensional environment, which has been used previously in the majority of the robotic rehabilitation techniques) and because it was designed specifically for human interaction (making it inherently safer than the converted manufacturing robots). The position of the fingertip was calculated using encoders attached to the motors of the robot. The force applied to the fingertip was determined from the commanded force output, which was verified with an external force sensor before the experiment.

\subsection{Procedure}

The subjects for the study were 17 healthy male and female adults between the ages of 19 and 31 . All were right handed and no subject was known to have any neurological disorder. We asked each subject to sit on the chair facing the computer monitor and the robot so that he/she was approximately $12 \mathrm{~cm}$ above and $46 \mathrm{~cm}$ in front of the screen (see Fig. 1). The subject's right arm was then placed on a side support, and his/her index finger was secured with a finger splint attached to the end of the robot so that the index finger could only be moved at the metacarpophalangeal joint as shown in Fig. 2. The finger's range of motion was limited by a hard stop imposed by the robot's force at the $0^{\circ}$ flexion point (position 1) and by a small post at the $90^{\circ}$ flexion point (position 2). The subject held a post with the remainder of his/her fingers to secure the hand position, and the subject's right arm and finger were shielded from view (not shown in the figure).

Each experimental run consisted of 70 trials, and each trial consisted of two samplings. For each sampling, subjects were asked to move their occluded index finger from position 1 toward position 2 to learn the mapping between forces issued tangentially to the finger's semicircular trajectory as well as the height on the visual feedback bar displayed on the computer screen that corresponded to this movement. At the end of each trial, subjects were asked to judge whether the mappings between two consequent samplings were the same or different (in some cases the second sampling's visual feedback may have been slightly distorted). Subjects were allowed to move their index finger freely as long as they sampled beyond a line drawn on the

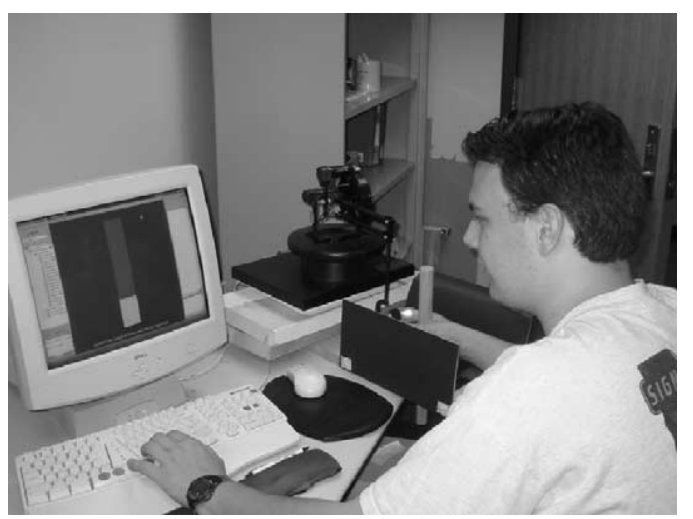

Fig. 1. Our experimental setup. A subject was seated on a chair facing a computer monitor that displayed the visual feedback bar of force/position. His index finger was secured to the robot with a finger splint. Forces were issued tangential to the finger's semicircular trajectory. The forearm was restrained to minimize shifting. The subject's view of his hand was occluded during the experiment with an opaque cover (not shown). 


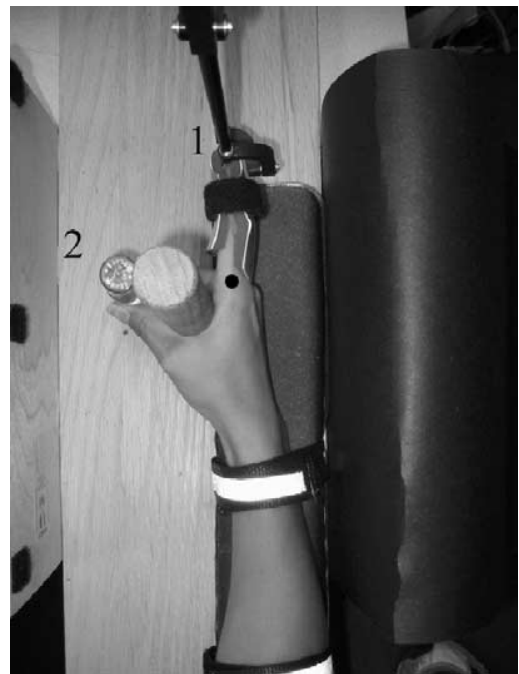

Fig. 2. The index finger was secured with a finger splint so that it could only be moved at the metacarpophalangeal joint (marked with $\bullet$ ). The subject held the post with the remainder of his/her fingers to secure the hand position. The finger's range of motion was limited by a hard stop imposed by the robot's force at the $0^{\circ}$ flexion point (position 1) and by a small post at the $90^{\circ}$ flexion point (position 2).

feedback bar between positions 1 and 2 at least once. After 5 $\mathrm{s}$, the bar turned from green to red to indicate that they had sampled for an adequate amount of time. Subjects, however, were encouraged to continue sampling the range of force until they felt comfortable with the mapping between the forces produced and the corresponding feedback bar height.

During each sampling, the robotic device produced forces on the finger pad that simulated a linear spring; these forces increased linearly with a Euclidean linear displacement in finger pad position beginning at position 1, and spring constants (ranging randomly between 30 and $80 \mathrm{~N} / \mathrm{m}$ ) determined their relationship. The spring constant stayed the same for the two samplings within one trial, but changed between trials. The Euclidean linear displacement between positions 1 and 2 varied from 78 to $98 \mathrm{~mm}$ depending on the finger length of the individuals. The extent of force being felt at any moment was mapped onto a continuous feedback bar height on the computer screen updated at $32 \mathrm{~Hz}$ (see Fig. 1). Because the force was linearly correlated with the finger position, the bar was also a positional indicator. The line drawn in the feedback bar (which the subjects were instructed to sample beyond) was randomized to match $30-40 \mathrm{~mm}$ of finger displacements from position 1 . The maximum height of the bar on the computer screen was $158.5 \mathrm{~mm}$, and the represented force and position at this point varied with the spring constant and visual distortion imposed. The finger position representation at this maximum height of the bar never exceeded $70 \mathrm{~mm}$ and the force representation never exceeded $5.6 \mathrm{~N}$. There were no markers within the bar to indicate the exact force or position of the finger.

Out of 70 trials, 30 trials featured no distortion between the first and second samplings and, therefore, during both trials the same mapping existed between force and height on the feedback bar. Ten trials featured a distortion of $10 \%, 10$ trials a distortion of $25 \%, 10$ trials a $40 \%$ distortion, and the remaining 10 trials a distortion of $55 \%$. A distortion of $x x \%$ means that the height, $h$, on the screen during the first sample was mapped as a height of $(1+x x / 100) h$ during the second sample. Each of the distortion percentage values was delivered with equal a priori probability.

Subjects were asked to judge the existence of distortion as well as to rate their confidence in their judgment on a scale of 1 to 5 . A rating of 1 indicated that the subject was extremely unsure whether or not his or her response was correct; whereas a rating of 5 indicated that the subject felt extremely confident that his/her response was correct. Subjects did not receive feedback regarding the accuracy of their judgments. In addition, because the spring constant changed from one trial to another, subjects could not rely on their memory from previous trials.

Fifteen of out 17 subjects voluntarily filled out questionnaires at the end of the experiment. These questionnaires were intended to identify strategies that the subjects might have used to determine feedback distortion.

\section{Results}

As expected, subjects noticed the existence of distortion in the second sampling more frequently when the distortion level was higher. Fig. 3 shows the percent of subjects' responses that claimed the existence of distortion with confidence plotted against the actual distortion level. The criterion set for claiming distortion with confidence was a rating of 3 or higher. With no distortion in the visual feedback, subjects claimed that the mapping changed $35.7 \%$ of the time, which means that they responded correctly $64.3 \%$ of the time (a random guess would produce the correct response $50 \%$ of the time). JNDs for both force and position were computed as

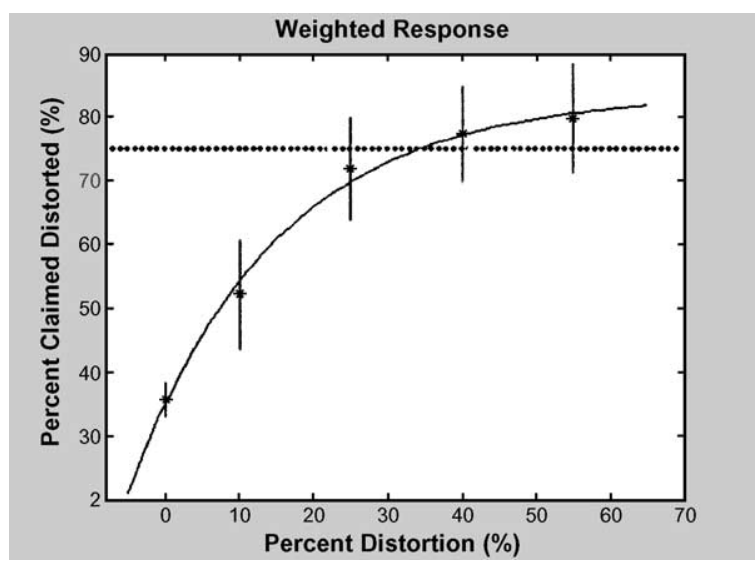

Fig. 3. The percentage of responses that subjects claimed was distorted with confidence plotted against the distorted percentage level. Fifty percent mark random guesses and $75 \%$ mark the level defined to be just noticeable. 

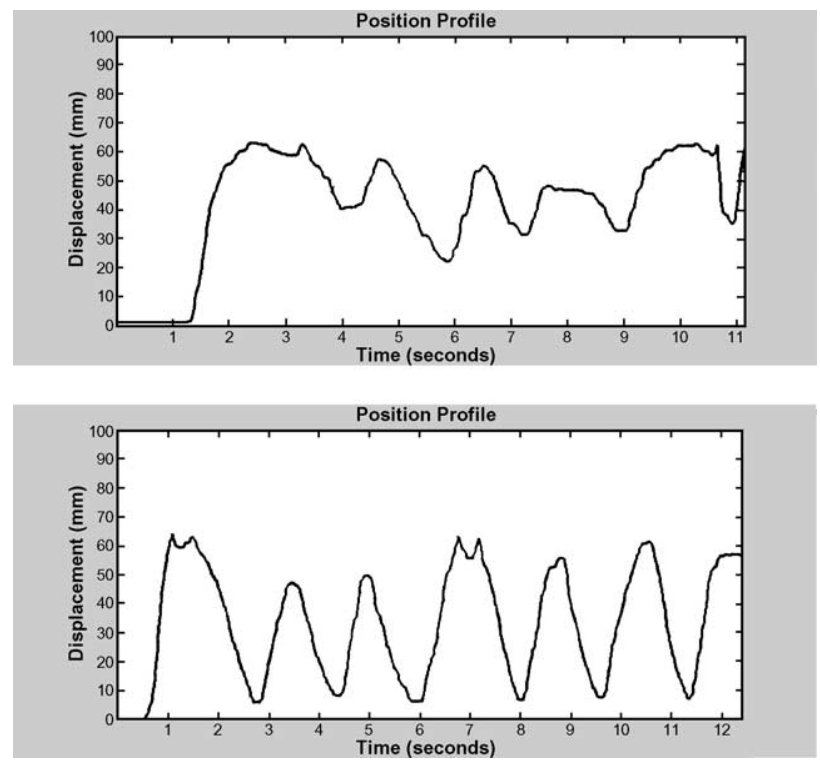

Fig. 4. Sampling strategies for different subjects. One subject moved his finger back and forth with larger amplitude than the other subject, but both subjects moved their fingers similarly for every trial.

the percentage increment in force that led to a sensitivity index of 1 , which occurs at $75 \%$ correct discrimination [10]. Therefore, we fit a function on the result to identify the exact percent distortion that was discriminated $75 \%$ of the time. We fit an exponential function of "percent correct respon$\mathrm{se}^{\prime \prime}=83.69-48.69 \mathrm{e}^{-(5.024 \times \text { percent distortion }) / 100}$ that achieved a correlation coefficient with true results of .993 . This exponential model predicted that subjects would achieve $75 \%$ accuracy, on average, when the distortion level was $35.9 \%$.

Because we provided freedom to identify the mapping in the sampling strategy, there was a wide range in sampling length and style. Two representative sampling movements are plotted in Fig. 4. All subjects moved their fingers back and forth around the threshold line specified on the screen. The minimum exploration time was $8.6 \mathrm{~s}$. The maximum exploration time was $38.5 \mathrm{~s}$, and the average exploration time was $19.3 \mathrm{~s}$. The style was consistent for each subject from trial to trial (average standard deviation in exploration length for each subject was $2.0 \mathrm{~s}$ ), but it was different from subject to subject (the standard deviation of the average of each subject was $4.2 \mathrm{~s}$ ). In addition, a correlation coefficient

Table 1

The responses (\%) of 15 subjects to a questionnaire entitled "Which part of the sampled range was used to determine the mapping?"

\begin{tabular}{lc}
\hline Used the whole range sampled & 33.3 \\
to determine the mapping & 60.0 \\
Used only the high end of the range & \\
$\quad$ and remembered just that & 6.7 \\
Used only the low end of the range & \\
$\quad$ and remembered just that & 40.0 \\
Used a spot in the range in between & low and high and remembered just that
\end{tabular}

Table 2

The responses (\%) of 15 subjects to a questionnaire entitled "Which component was used to compare mapping with visual feedback?"

\begin{tabular}{ll}
\hline Used the finger position & 80.0 \\
& position alone: 6.7 \\
& 86.7 \\
Used the force felt on the finger & force alone: 20.0 \\
& 53.3 \\
Used both force and position & both position and force: $66.7 \%$ \\
information at the same time &
\end{tabular}

relating sample time and confidence value was computed over trials for each subject. The mean correlation coefficient was -.14 , and the variance among the terms was .018 . Notably, 14 of the 17 subjects generated negative correlation coefficients, indicating, on average, a relationship between low confidence and lengthy sampling times.

Tables 1 and 2 show the responses to the questionnaire that 15 subjects filled out voluntarily at the end of the experiment. Subjects were allowed to mark more than one response if they had used more than one strategy during the experiment. Despite the fact that subjects moved their fingers back and forth as if they were using the whole range of force that they felt on their fingers, $60.0 \%$ of subjects responded that they tried to remember the highest point they reached. When they were asked which component they used to compare mapping with visual feedback, some subjects checked both the force and position boxes but did not check that they used both at the same time. When these subjects were added to the group using both force and position, $66.7 \%$ of the subjects used both position and force.

\section{Discussion}

Our results indicate that distortions in the visual representation of force production and position displacement are tolerated beyond the bounded JNDs for force and position in our virtual environment. Specifically, the force and position JNDs in our previous study were $14.4 \%$ and $18.0 \%$, respectively, while subjects tolerated inaccuracies in visual representation of force and position up to $35.9 \%$ in our present experiment.

JND is defined as the percentage of increase in stimulus that is required to reliably distinguish two stimuli. Strictly speaking, we did not increase the force or position for the second stimulus in a trial, but we did change the mapping of the visual representations for the second stimulus in a trial. We believe that the faculties involved in detecting distortions in the visual representations of force/position are not identical to those involved in detecting force/position augmentation without visual feedback. Therefore, we do not claim that we changed the force and position JND defined by the definition above, but we do claim that we were able to alter the ability of subjects to distinguish two stimuli using distorted visual representations in the virtual environment. This is an important result because it indicates that 
subjects, with inaccurate visual feedback, are willing to believe visual representations beyond the JNDs defined for both force and position.

Because force and position information were correlated in our experiment, subjects could rely on the information with the smaller JND to detect the difference between the actual position/force and its visual representation. If there was no visual feedback distortion, we expected to see a JND close to $14 \%$, which is the JND for force in our virtual environment. Therefore, we extended the JND of force by over three times with distorted visual representations.

For similar finger movements, previous literature indicates that JNDs were found to be relatively constant between 2.5 and $10 \mathrm{~N}$ [9]. When subjects sampled the mapping between the visual representations and the actual force and position, they moved their fingers back and forth within a range, on average, between 25 and $62 \mathrm{~mm}$ Euclidean distance, which corresponded to $0.75-4.96 \mathrm{~N}$. As indicated in the questionnaire, to determine the existence of distortion, $60 \%$ of the subjects indicated that they used the highest point they reached and tried to remember which point that was and how the force/position felt on their finger at that point. Because the visual distortion was determined using a percentage rather than a constant addition to the entire display, there should have been no advantage in picking a higher point rather than a lower point sampled, if JNDs are indeed constant. In addition, since subjects did not reach anywhere near the top of the display bar or the physical limit of the finger, there should have been no advantage in remembering a high position on the bar. The fact that subjects preferred to use large forces for their discrimination task indicates that JNDs may be larger for small forces, especially forces below the range identified to have constant JND, and this issue should be explored further before implementing a therapeutic paradigm for people recovering from strokes who may only be capable of producing a small amount of force.

The ability to extend JNDs using distorted visual representations in the virtual environment is encouraging for purposes of stroke rehabilitation. In order to extend the strength and mobility of people recovering from strokes, it is important to create an environment that can steer them towards producing more force and moving further than they think they are capable of doing. To get them to do more, we can use visual feedback that indicates that they are doing less than they are actually doing and, thus, encourage them to train their strength and mobility at a level beyond what they believe to be possible. In a different application, we can also use this distortion as a motivational factor. By distorting visual feedback to indicate that they are doing more than they are actually doing, we may encourage them to have higher expectations for themselves. With this large perceptual gap for force and position induced by distorted visual feedback, we can produce a wide range of imperceptible distortions between visual representations and actual force production and positional displacement.

For this experiment, subjects were told specifically to focus on the disagreement between the actual and the virtual information. We believe that if subjects are not told that there may be a discrepancy between their actual and virtual movements, we would be able to extend this perceptual gap even further. As our next step, we are interested in testing whether this large perceptual gap can be used to steer individuals towards producing more or less force, or moving shorter or longer distances, without being aware that they are doing so.

\section{References}

[1] Taub E. Overcoming learned nonuse: a new behavioral medicine approach to physical medicine. In: Carlson JG, Seifert SR, Birbaumer N, editors. Clinical applied psychophysiology. New York: Plenum; 1994. p. $185-220$.

[2] Aisen ML, Krebs HI, Hogan N, McDowell F, Volpe BT. The effect of robot-assisted therapy and rehabilitative training on motor recovery following stroke. Arch Neurol 1997;54(4):443-6.

[3] Krebs HI, Volpe BT, Palzzolo J, Rohrer B, Ferraro M, Fasoli S, et al. Robot-aided neuro-rehabilitation in stroke: interim results on the follow-up of 76 patients and on movement performance indices. In: Mokhtari M, editor. Integration of assistive technologies in the information age. Paris: IOS Press; 2001. p. 45-59.

[4] Kahn LE, Averbuch M, Rymer WZ, Reinkensmeyer DJ. Comparison of robot-assisted reaching to free reaching in promoting recovery from chronic stroke. In: Mokhtari M, editor. Integration of assistive technologies in the information age. Paris: IOS Press; 2001. p. 39-44.

[5] Lum P, Burgar C, Shor P, Majmundar M, Van der Loos M. Robotassisted movement training compared with conventional therapy. Arch Phys Med Rehab 2002;83:952-9.

[6] Ross HE, Murray DJ, Weber EH. The sense of touch. New York: Academic Press; 1978.

[7] Brodie E, Ross H. Sensorimotor mechanisms in weight discrimination. Percept Psychophys 1984;36:477-81.

[8] Jones LA. Matching forces: constant errors and differential thresholds. Perception 1989;18(5):681-7.

[9] Pang X, Tan HZ, Durlach N. Manual discrimination of force using active finger motion. Percept Psychophys 1991;49(6):531-40.

[10] Allin S, Matsuoka Y, Klatzky R. Measuring just noticeable differences for haptic force feedback: a tool for rehabilitation. Haptic Symp 2002; 299-303. 